für sein zukunftweisendes Konzept und sein nachhaltiges Angebot prämiert. LEO-BW ist Preisträger des bundesweit ausgetragenen Innovationswettbewerbs "365 Orte im Land der Ideen" (http:// www.land-der-ideen.de/365-orte/365-orte-im-landideen). Unter über 2.000 Bewerbern wurde das Projekt von einer namhaften Jury als einer der 365 Orte für das Jahr 2012 ausgewählt.

"Deutschland - Land der Ideen“ ist eine gemeinsame Initiative von Wirtschaft und Bundesregierung und prämiert in Kooperation mit der Deutschen Bank seit 2006 Ideen und Projekte, die einen nachhaltigen Beitrag zur Zukunftsfähigkeit Deutschlands leisten.

\section{Fazit \& Ausblick}

„Baden-Württemberg hat ein Gedächtnis: Das landeskundliche Portal LEO-BW geht online" - So hieß es in der Pressemitteilung des Ministeriums für Wissenschaft, Forschung und Kunst vom 25.04.2012 (Nr. 58/2012). Als offizieller Beitrag der Landesregierung wurde LEO-BW zum Landesjubiläum 2012 der Öffentlichkeit präsentiert. Mit diesem werden bereits vorhandene Datenbanken und Informationssysteme der beteiligten Institutionen in normierter und standardisierter Form vernetzt.
Es bietet Bürgerinnen und Bürgern, wissenschaftlich Forschenden, Schülerinnen und Schülern sowie allen Interessierten mit wenigen Klicks und "aus einer Hand" umfassende Informationen zu Baden-Württemberg, seinen Persönlichkeiten und Orten und damit einen erheblichen Mehrwert gegenüber der Einzelinformation.

In äußerst kurzer Projektzeit wurde damit ein bisher in Deutschland einzigartiges Projekt umgesetzt. Doch LEO-BW ist auf Langfristigkeit und Nachhaltigkeit angelegt. So gingen die Arbeiten auch nach Freischaltung von LEO-BW für die Öffentlichkeit weiter. Neben der Datenpflege der Altbestände und der Verbesserung der ErschlieBungsleistung mit Normdaten wurden weitere Systemoptimierungen durchgeführt. Die Kooperationspartner gewährleisten die Aktualität ihrer Inhalte durch regelmäßige Datenimporte. Für eine zweite Projektphase sind außerdem die Einbindung ergänzender Inhalte von aktuellen und neuen Partnern, Web 2.0-Funktionen und Angebote für spezielle Zielgruppen, beispielsweise im Bildungsbereich, geplant.

Anja Thalhofer

\title{
200 Jahre Bibeln aus Stuttgart Zum Jubiläum
} der Württembergischen Bibelanstalt / Deutschen Bibelgesellschaft

Die Übersetzung und Verbreitung der Bibel ist motiviert von der Überzeugung, dass „der Glaube aus der Predigt, das Predigen aber durch das Wort Christi" kommt (Römerbrief 10,17). So entstehen bereits vor der Reformation vierzehn hochdeutsche und vier niederdeutsche Bibelausgaben. Diese fußen allerdings auf der lateinischen Vulgata, nicht auf den biblischen Grundtexten. Martin Luthers Bibelübersetzung, die zwischen 1522 (Septembertestament: nur Neues Testament) und 1534 (erste Vollbibel) entsteht und in einer letzten von inm bearbeiteten Version 1545 als "Ausgabe letzter Hand" erscheint, geht unter Einfluss des Humanismus von den biblischen Ursprachen aus. Die Reformation betreibt den Transfer des philo- logischen „Ad fontes" („Zurück zu den Quellen“) der Humanisten in die theologische Erkenntnistheorie. So geht es fortan um einen möglichst direkten, authentischen Zugang des Individuums zum Wort Gottes - ohne normierenden Rückbezug auf die Tradition oder das kirchliche Lehramt. Die Bibel soll unter das Volk kommen und zwar in möglichst verständlicher und wirksamer Weise. Zu den vier für die Reformation charakteristischen Exklusivformeln gehört auch das "Allein die Schrift" (Sola Scriptura).

Diese Zentrierung des Glaubens auf die Bibel im Protestantismus führt in der Folgezeit zu einem Aufblühen des Buchdrucks gerade im Bereich der 
Bibelproduktion. Davon profitiert auch die katholische Bevölkerung, weil in Reaktion auf die Lutherbibel in Deutschland schnell dezidiert katholische Ausgaben auf den Markt gebracht werden. Die geringe Verbreitung der Lesefertigkeit, vor allem aber der hohe Anschaffungspreis der häufig prachtvoll ausgestatteten Bibeln schränkt die Zielgruppe der Bibelrezeption faktisch jedoch auf die gesellschaftliche Elite bzw. Institutionen ein. Erst der Pietismus betont das persönliche Glaubensleben mit Bekehrung und Heiligung sowie die Berufung der Laien im kirchlichen Bereich so stark, dass der Wunsch nach einfachen, preisgünstigen Bibelausgaben für breite Bevölkerungsschichten unabweisbar wird.

Ein erster Anlauf zur Massenproduktion von Bibeln wird mit der 1710 gegründeten Cansteinschen Bibelanstalt in Halle unternommen. Die wichtigste technische Neuerung ist der Stehsatz: Die Lettern werden nicht für jeden Bogen neu gesetzt, sondern bleiben für sämtliche Bögen erhalten, um dadurch in kurzen Abständen weitere Auflagen drucken zu können.

Die Bibelgesellschaften werden zu einer echten Konkurrenz für die Verlage der Bibelausgaben klassischen Stils (z.B. Endter, Nürnberg), jedoch erst mit dem Beginn der Erweckungsbewegung Anfang des 19. Jahrhunderts. Zur wichtigsten Bibelgesellschaft wird neben Canstein die 1812 in Stuttgart gegründete Württembergische Bibelanstalt. Sie ist über die Person ihres Initiators Karl Friedrich Adolf Steinkopf (1773 - 1859) vermittelt, ein Ableger der seit 1804 bestehenden British and

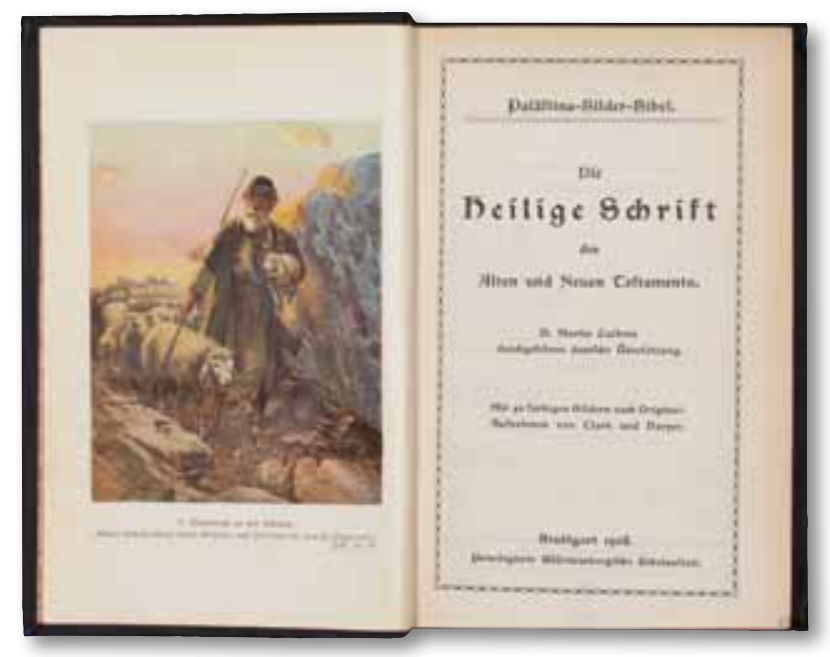

B deutsch 190801 : Palästina-Bilder-Bibel
Foreign Bible Society, London. Steinkopf knüpft im Auftrag der Briten Kontakte auf dem Kontinent und gewinnt in Württemberg weitere Interessenten. Die fünfzehn an der Gründung beteiligten Personen verständigen sich auf das Anliegen, „die Verbreitung der Bibel unter den ärmeren Volksklassen im evangelischen Württemberg" zu fördern. Dafür erhält die Bibelanstalt 1813 das königliche Privileg in Gestalt der Portofreiheit, ab 1882 durch einen staatlichen Zuschuss. 1815 kann die erste Lutherbibel in einfacher Form - eigentlicher Bibeltext mit wenigen Parallelstellen und kurzen Summarien - in einer Auflage von 10.000 Exemplaren erscheinen. In kurzer Abfolge wird diese Ausgabe mit jeweils kleinen Verbesserungen immer wieder nachgedruckt.

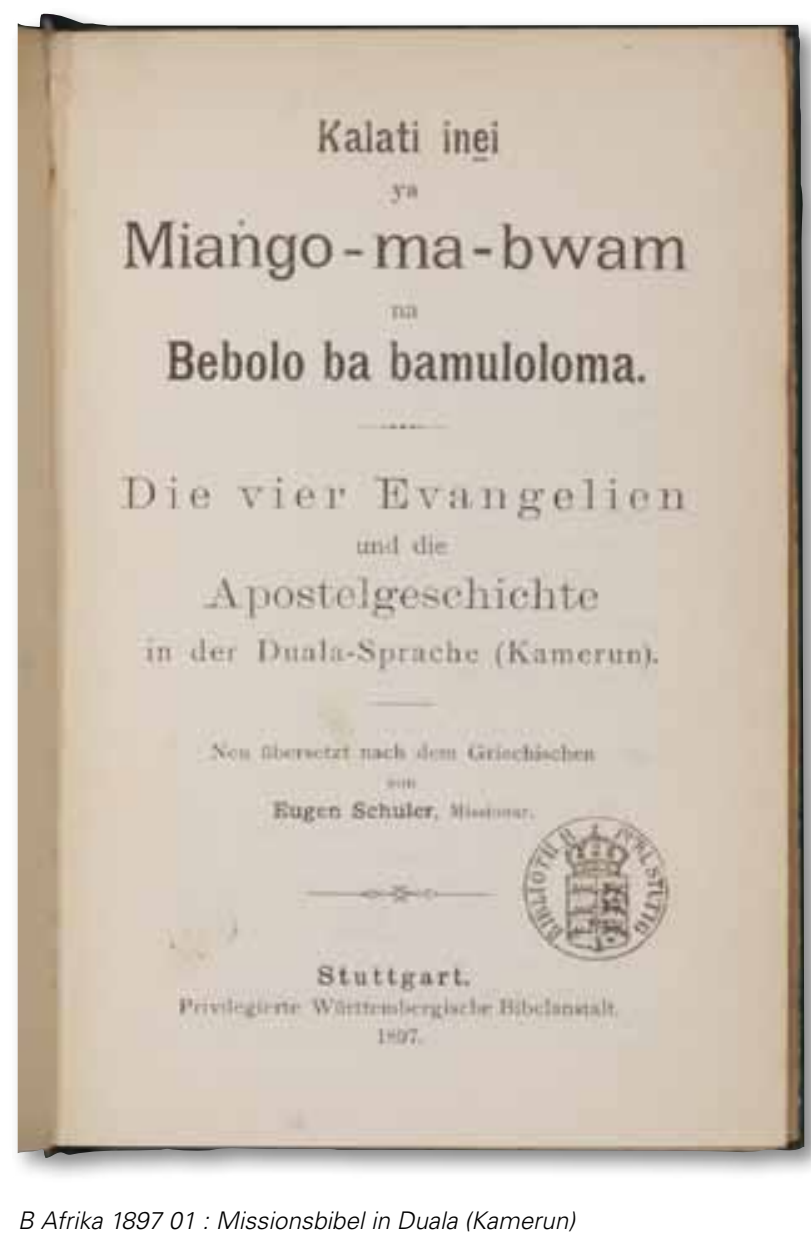

B Afrika 189701 : Missionsbibel in Duala (Kamerun)

Die Württembergische Bibelanstalt spiegelt die Textstufen der 1892, 1912, 1956/1964, 1975, 1984 revidierten Lutherbibel wider. Im 19. Jahrhundert erlaubt die Einfachheit einer Bibelausgabe einen hohen Rezeptionsgrad. In der weiteren Entwicklung der Bibelproduktion entstehen allerdings reicher ausgestattete Bibeln. Das Bild wird als Haftpunkt einer ganzheitlichen Begegnung mit 
dem Wort Gottes erkannt. Die sogenannte Palästina-Bilder-Bibel von 1908 ist die erste mit zahlreichen Illustrationen ausgestattete Ausgabe der Württembergischen Bibelanstalt.

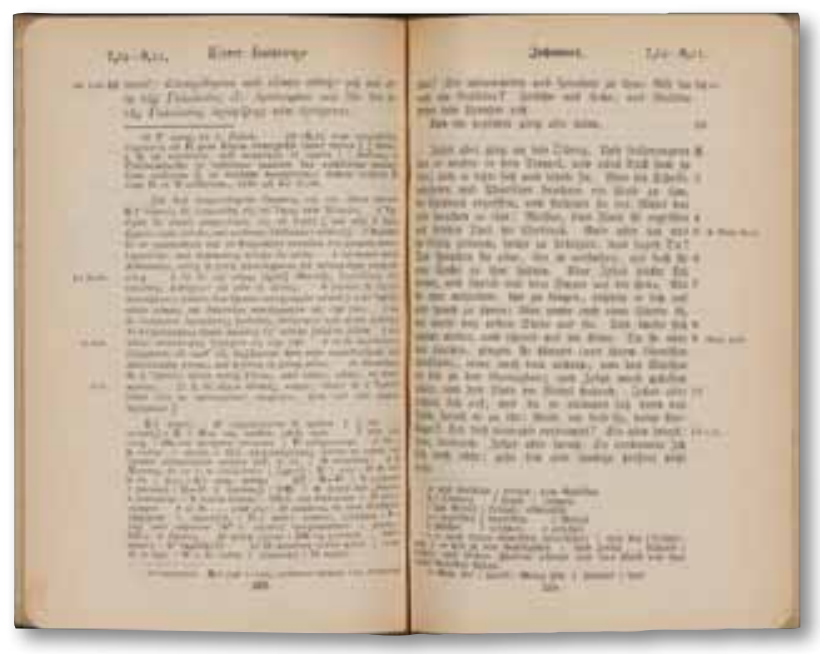

B griech. 189881 : Griechisch-deutsche Textausgabe ]

Die Komplexität der Gesellschaft wie der Wechselfälle des Lebens findet ihren Ausdruck in einer wachsenden Zahl variierender Bibelausgaben. Das gilt für Zielgruppen wie Blinde, Kinder und Jugendliche. Beigaben zum Bibeltext ermöglichen in Bibeln zu bestimmten Anlässen wie z.B. Konfirmation oder Trauung eigene Einträge. Moderne Bibelübersetzungen erleichtern Menschen, denen Luthers Deutsch trotz aller Revision zu schwerfällig oder zu theologisch profiliert ist, den Zugang zu den biblischen Inhalten.

Seit den 1890er Jahren erscheinen in Stuttgart Bibeln in den Sprachen der missionierten Völker. Dies wird nach dem Ende der Kolonialzeit weiter- geführt und mündet in die 1975 begonnene "Aktion Weltbibelhilfe".

An den biblischen Urtextausgaben aus Stuttgart führt kein Weg vorbei. Ab 1898 bzw. 1925 erscheinen hier die maßgeblichen textkritischen Editionen des griechischen Neuen bzw. hebräischen Alten Testaments.

Mit einer Zwischenstation als Teil der 1976 gegründeten Deutschen Bibelstiftung firmiert die Württembergische Bibelanstalt durch Zusammenschluss mit dem Evangelischen Bibelwerk seit 1981 als „Deutsche Bibelgesellschaft". Die „Württembergische Bibelgesellschaft" widmet sich der bibelmissionarischen Arbeit. Die Namensänderung unterstreicht das Gewicht des Bibeldrucks in Stuttgart. Dieser ist über 200 Jahre hinweg mit Kontinuität in Motivation und Engagement geschehen.

Aus diesem Anlass findet in der Württembergischen Landesbibliothek vom 26. September bis 29. Dezember 2012 eine Ausstellung statt. Fast alle ausgestellten Bibelexponate stammen aus der umfassenden Bibelsammlung der Landesbibliothek. Druck, Verbreitung, Dokumentation und Erforschung von Bibeln sind durch das kooperative Zueinander von Württembergischer Bibelanstalt bzw. Deutscher Bibelgesellschaft und Württembergischer Landesbibliothek seit 200 Jahren in Stuttgart verzahnt. Die Ausstellung mit begleitendem Katalog ermöglicht einen Überblick zu dieser Entwicklung.

\section{Kinderbücher des Ersten Weltkriegs aus den Beständen der Bibliothek für Zeitgeschichte}

\section{Einleitung}

Unter den erhaltenen Büchern aus der ehemaligen „Kriegssammlung" des Ersten Weltkrieges, die der schwäbische Industrielle Richard Franck 1915 in Berlin aufbaute und die heute den Grundstock der Sammlung der Bibliothek für Zeitgeschichte (BfZ) bildet, finden sich auch zahlreiche Kinderbücher aus dem Ersten Weltkrieg. 